\title{
Análisis financiero en las empresas del sector servicios en Ecuador entre los años 2016-2017
}

\author{
Paredes Venegas, Cristina Alexandra* \\ Chicaiza Chicaiza, Bryan Leandro* \\ Ronquillo Llundo, Joselyn Tatiana* \\ *Universidad de las Fuerzas Armadas ESPE \\ * Carrera de Licenciatura en Finanzas y Auditoría \\ E-mail: caparedes16@espe.edu.ec
}

Recibido: 17 julio de 2019 Aprobado: 23 diciembre de 2019

\begin{abstract}
Resumen
En el presente trabajo se muestra los indicadores financieros del sector servicios del Ecuador. Los indicadores financieros son medidas que ayudan en el análisis del estado de la empresa desde un punto de vista individual, comparativamente con la competencia. La información se obtuvo a partir de la superintendencia de compañías, de la cual se calculó a partir de la información obtenía en los estados financieros como son el estado de situación financiera y estado de resultados de los años 2016 y 2017. Los indicadores utilizados nos permitirán conocer la situación en las que se encuentra el sector servicios según el análisis que se realice de los indicadores de liquidez, actividad, rentabilidad y endeudamiento.
\end{abstract}

Palabras claves: Indicadores, Liquidez, Actividad, Rentabilidad, Endeudamiento 


\title{
Analysis financial in the companies of the services sector in Ecuador between the years 2016-2017
}

\begin{abstract}
In the present work, the financial indicators of the services sector of Ecuador are shown. Financial indicators are measures that help in the analysis of the state of the company from an individual point of view, comparatively with the competition. The information was obtained from the superintendence of companies; it was calculated from the information obtained in the financial statements as the statement of financial position and the income statement for the years 2016 and 2017. The situation in which It finds in the services sector according to the analysis that is made of the indicators of liquidity, activity, profitability and indebtedness.
\end{abstract}

Keywords: Indicators, Liquidity, Activity, Profitability, Indebtedness.

\section{Introducción}

Este estudio se enfoca en el análisis financiero aplicado a las empresas del sector servicios en el Ecuador dentro del periodo 20162017, con la finalidad de conocer a través de los indicadores financieros su liquidez, el uso de sus recursos, rentabilidad y endeudamiento. En contexto con lo antes mencionado los indicadores financieros según Ibarra (2009), los primeros estudios que existen sobre estos indicadores se remota tras la gran depresión de 1929, se les atribuye a dos banqueros norteamericanos que bajo la supervisión de Alexander Wall utilizaron como una técnica de gestión, consistía en un análisis dirigido hacia los clientes potenciales utilizando estos indicadores, por otra parte, de acuerdo con Torres, M. (2014) durante el sigo $\mathrm{XX}$ se estandarizan dichos indicadores creando niveles óptimos para las razones financieras, sin embargo puesto que dentro de la estandarización no se tomo en cuenta la existencia de múltiples organizaciones no se puedo lograr dicha estandarización.

El estudio se enfoca en el sector servicios el cual está considerado dentro de la economía como el sector terciario es el encargado de realizar actividades enfocadas a satisfacer necesidades, su característica principal es ser intangible así también es uno de los sectores más importantes del país pues es la mayor fuente de empleo por otra parte, es el sector más grande del país y es el segundo sector con mayor ingresos producto de sus operaciones.

Es por lo antes mencionado que se ha procedido a realizar una investigación a este sector y mediante el uso de métodos estadísticos los cuales permiten seleccionar una muestra significativa a fin de poder analizar dichos datos y conocer a través de los indicadores financieros. Para ello se recurrió a la recopilación bibliográfica documental, en distintas bases teóricas a fines al tema de investigación y dentro del portal de información que proporciona la Superintendencia de Compañías y Seguros del Ecuador.

\section{Sector servicios}

De acuerdo con Herrera (2014), el sector servicios es unión de varios procesos y actividades con el objeto de satisfacer necesidades de la población a través de la prestación de servicios.

Paredes Venegas, Cristina Alexandra; Chicaiza Chicaiza, Bryan Leandro \& Ronquillo Llundo, Joselyn Tatiana 
Así también concuerda Barrera \& Jiménez (2017), puesto que lo definen como un sector el cual cumple una serie de actividades que van desde servicios profesionales, seguridad, administrativos, entre otros por otra parte son de vital importancia en el desarrollo de una economía puesto que el continuo crecimiento de estas va de la mano con el empleo y la competitividad de las empresas en el sistema.

De la misma manera señala Torres (2006), que son todas las actividades cuya característica central es la intangibilidad, es un conjunto de actividades que está enfocada a atender las demandas tales como apoyo administrativo, hotelería entre otros.

En base a lo antes expuesto el sector de servicios dentro de la economía pertenece al sector terciario el cual comprende actividades enfocadas al desarrollo y a la satisfacción de las necesidades de la población, así como de organismos y entes, por otra parte, dentro de las características principales que marcan a este sector es que el producto de estos no es tangible.

\section{Empresas del sector servicios en Ecuador}

Dentro del Ecuador según el Instituto Nacional de Estadísticas y Censos (INEC, 2016) el número de empresas que actualmente tiene el país son 843745 entre las cuales figuran activas y no activas. El sector servicios es uno de los sectores más grandes del país, puesto que alberga alrededor de 342000 empresas entre activas y no activas lo que representa el $49,59 \%$ del total de empresas dentro de ellas comprende actividades tales como: Suministro de Electricidad, Gas, Vapor Agua, Alcantarillado, Servicios de Comida, Información y Comunicación y otros servicios. De estas la mayoría tienen localidad entre Pichincha y Guayas por otra parte en cuanto a la participación en ventas ocupan el segundo lugar con ventas de 37.400 millones de dólares por año esto representa un $25,32 \%$ del total de ventas de empresas existentes en el país.

\section{Tabla 1}

Número de empresas por Sectores

\begin{tabular}{|l|r|r|}
\hline Sector Económico 2016 & No de Empresas & Porcentaje \\
\hline Servicios & 342488 & $40,59 \%$ \\
\hline Comercio & 308956 & $36,62 \%$ \\
\hline Agricultura, ganadería, silvicultura y pesca & 87926 & $10,42 \%$ \\
\hline Industrias Manufactureras & 72735 & $8,62 \%$ \\
\hline Construcción & 28678 & $3,40 \%$ \\
\hline Explotación de Minas y Canteras & 2962 & $0,35 \%$ \\
\hline Total & 843745 & $100 \%$ \\
\hline
\end{tabular}

Fuente: Recuperado de Instituto Nacional de Estadísticas y Censos, 2016 
Esto a su vez representa cierto beneficio para la población y el desarrollo económico pues del total de estas empresas, el sector servicios fue la mayor fuente de empleo del total de entidades en el país, con un registro del personal ocupado como afiliado del $54,94 \%$ lo que representa $1 ` 613.763$ personas.

\section{Tabla 2}

Participación del personal afiliado según sector económico

\begin{tabular}{|l|r|r|}
\hline Sector Económico 2016 & No de Empresas & \multicolumn{1}{|l|}{ Porcentaje } \\
\hline Servicios & 342488 & $40,59 \%$ \\
\hline Comercio & 308956 & $36,62 \%$ \\
\hline Agricultura, ganadería, silvicultura y pesca & 87926 & $10,42 \%$ \\
\hline Industrias Manufactureras & 72735 & $8,62 \%$ \\
\hline Construcción & 28678 & $3,40 \%$ \\
\hline Explotación de Minas y Canteras & 2962 & $0,35 \%$ \\
\hline Total & 843745 & $100 \%$ \\
\hline
\end{tabular}

Fuente: Recuperado de Instituto Nacional de Estadísticas y Censos, 2016

\section{Análisis financiero}

De acuerdo con Madrigal, Ayala. \& Chávez (2015), es un proceso el cual se enfoca en el análisis del momento en el que se encuentra una entidad en cuanto a la parte financiera, esta puede ser presente o pasada y a su vez el análisis de los resultados producto de sus operaciones, este análisis nos muestra si se están o no cumpliendo los objetivos, para si identificar fortalezas y corregir fallas.

De acuerdo con Prieto (2010), se puede señalar como un proceso que comprende recolectar, interpretar y comparar los estados financieros en periodos distintos $o$ en un solo periodo, esto se da a través de cálculo de porcentajes, tasas, indicadores a fin de evaluar el desempeño.

En cuanto al criterio de Bravo (2017), menciona que entre los beneficios de la realizar el análisis financiero se puede determinar el ambiente interno en el cual se desenvuelve la empresa así también se puede analizar las decisiones que toma la empresa con respecto al entorno externo con el fin de tomar las mejores estrategias que sean favorables para la empresa

De acuerdo con Prieto (2010), se puede realizar de manera horizontal y vertical. En lo que respecta al análisis vertical consiste en tomar un estado financiero de un solo año y se relaciona en porcentajes con un total determinado, se considera estático puesto que estudia la situación en momento determinado.

Por otra parte, el análisis horizontal es dinámico, ya que toma en cuentas las cambios que han sufrido las cuentas en un periodo determinado, y según Puerta, Vergara, \& Huertas (2018) esto a su vez permite estimar tendencias y efectuar proyecciones.

Paredes Venegas, Cristina Alexandra; Chicaiza Chicaiza, Bryan Leandro \& Ronquillo Llundo, Joselyn Tatiana 


\section{Razones financieras}

Según Prieto (2010), es la relación numérica entre dos cuentas ya sea del estado de situación financiero o el de resultados, estas razones a su vez permiten identificar puntos débiles o fuertes. Por su parte Madrigal, Ayala \& Chávez (2015), concuerdan en la importancia de estudiar estas razones y a su vez señala que son sencillas medidas, las cuales son objeto de análisis y estudio, se clasifican en cuatro grupos:

\section{Razones de liquidez}

Según Galán, Castro \& Pérez (2016), se miden si existe la cantidad necesaria de recursos a fin de poder constatar si se puede cubrir las deudas en el corto plazo Así concuerda Prieto (2010), pues afirma que es medir que tan capaz es la empresa de cumplir sus obligaciones en menos de un años, convirtiendo sus activos en efectivo en el plazo no mayor de un año. Esto implica analizar la disponibilidad del activo la velocidad con que se transforma en líquido y la exigibilidad de las deudas.

\section{Razones de solvencia o endeudamiento}

Por su parte la Superintendencia de Compañías (s.f) define como indicador de solvencia una razón mediante la cual se mide que porcentaje tienen de participación los acreedores dentro del financiamiento que posee la empresa a fin de determinar su riesgo, según Puerta, Vergara \& Huertas (2018), señala que mide que tan capaz es la empresa de cubrir necesidades en el largo plazo.

\section{Razones de actividad}

Según Prieto (2010), afirma que nace producto del principio financiero el cual dice que los activos deben contribuir al logro de los objetivos, no es productivo tener activos innecesarios. En este sentido la Superintendencia de Compañías (s.f) lo define como un indicador el cual mide con qué frecuencia la empresa usa sus recursos y su nivel de eficiencia en cuanto a la recuperación en términos monetarios.

\section{Razones de rentabilidad}

Los indicadores de rentabilidad de acuerdo con Puerta, Vergara \& Huertas (2018) mide cuan capaz la empresa genera ganancias para distribuir a los socios. Se mide el resultado producto de las actividades luego de pagar las obligaciones con terceros con relación a los ingresos, capital o patrimonio (Galán, L. Castro, M. \& Pérez, L., 2016, p. 3715). Mide la efectividad de la administración de empresa de controlar costos y gastos, la Superintendencia de Compañías (s.f).

\section{Estados financieros}

Según Núñez (2015), señala que los estados financieros son documentos elaborados al final del ciclo contable los mismos que sirven para conocer los resultados producto de las transacciones propias de la actividad de la empresa, así también es un instrumento a treves del cual se puede analizar y tomar decisiones, cabe señalar tanto su estructura y elaboración se debe regir a las normas internacionales de información financiera emitidas por el IASB.

Por su parte Zhanay (2013), menciona que los estados financieros son un representación de la estructura de en cuanto a las composición de las operaciones realizadas por la empresa, 
su objetivo es brindar información verdadera, para así poder brindar informes de situación financiera en una fecha determinada en cuanto a los resultados producto del giro del negocio.

De acuerdo con el criterio de Panchi (2017), la información financiera que revela cada entidad debe contener atributos los cuales deben ser objetivos, tener homogeneidad $\mathrm{y}$ ser comprables en cualquier momento, todo esto aplicando las normas internacionales de información financiera, para que puedan ser sometidas a análisis los mismos que luego servirán para tomar decisiones

Con respecto a lo antes mencionado cabe destacar que los estados financieros son de gran importancia puesto que son parte fundamental dentro de la empresa pues reflejan los movimientos que la empresa ha incurrido durante un periodo de tiempo. También es importante debido a que a través del análisis financiero permite seleccionar decisiones correctas en cuanto a inversión y crédito, para lo cual se debe conocer la estructura de la empresa, su capacidad económica, a fin de analizar la solvencia y liquidez de la empresa

\section{Metodología}

La metodología que se utilizó en la elaboración del presente es cuantitativa, la misma que tiene como objetivo principal el análisis financiero de las composiciones de los estados financieros presentados por las empresas pertenecientes al sector servicios.

Baena (2014), dice que "La investigación documental es la búsqueda de una respuesta específica a partir de la indagación en documentos." (p.12). En base a lo antes mencionado se puede definir como aquel proceso el cual toma información secundaria como parte fundamental de la investigación.

Morales (2014), concuerda que la investigación bibliográfica documental es producto de una serie de procesos secuenciales en los cuales se recurre a documentos escritos o fuentes previamente ya abordadas por otros autores mismos que deben ser analizados por la persona que realiza la investigación a fin de poder sintetizar la información.

Según los criterios de los autores la investigación bibliográfica documental es aquella que nos permite recolectar información de fuentes fidedignas. Para nuestra investigación recurrimos a la búsqueda de información confiable como reportes, estados financieros, directorio de las empresas que reportaron información a la Superintendencia de Compañías y Seguros.

Según Hernández (2014), en su seminario para tesis define a la población como:

Dentro de la investigación es importante establecer cuál es la población y si de esta se ha tomado una muestra, cuando se trata de seres vivos; en caso de objetos se debe establecer cuál será el objeto, evento o fenómeno a estudiar. Se entiende por población al conjunto total de individuos, objetos o medidas que poseen algunas características comunes observables en un lugar y en un momento determinado. (p.2)

En base a lo antes expuesto se recolecto información para la muestra misma cuya población fueron las empresas activas registradas

Paredes Venegas, Cristina Alexandra; Chicaiza Chicaiza, Bryan Leandro \& Ronquillo Llundo, Joselyn Tatiana 
dentro de la Superintendencia de Compañías categorizadas como N, Actividades de Servicio Administrativo y de Apoyo y S, Otros Servicios

\section{Tabla 3}

Empresas Activas Sector Servicios

\begin{tabular}{|l|l|r|}
\hline Clasificación & Actividad & Número de empresas \\
\hline $\mathrm{N}$ & Actividades de Servicio Administrativo y de Apoyo & 5786 \\
\hline $\mathrm{S}$ & Otros Servicios & 504 \\
\hline Total & & 6290 \\
\hline
\end{tabular}

Fuente: Recuperado de Superintendencia de Compañías de Ecuador
Tomando en cuenta el trabajo de Hernández, Fernández \& Baptista (2014), en su libro de Metodología de la Investigación sintetizan a la muestra como:

La muestra es un subgrupo de la población de interés sobre el cual se recolectarán datos, y que tiene que definirse $y$ delimitarse de antemano con precisión, además de que debe ser representativo de los resultados encontrados en la muestra se generalicen o extrapolen a la población. (...). El interés es que la muestra sea estadísticamente representativa. (p.173)

En consecuencia, Vargas (2008), señala que el muestreo aleatorio simple "Es un muestreo en el cual se asignan un número a cada elemento de la población y a través de algún medio mecánico se eligen tantos sujetos sean necesarios para completar el tamaño de la muestra requerida". (p. 38)

Para determinar el tamaño de la muestra según Vargas, R. (2008) señala que la muestra debe ser representativa pues a partir de esta se la población. El investigador pretende que mismos que tienen un total de empresas. estima valores equivalentes poblacionales para lo cual se utilizó la siguiente fórmula:

$$
n=\frac{\frac{Z^{2} * p(1-p)}{e^{2}}}{1+\left(\frac{Z^{2} * p(1-p)}{e^{2} N}\right)}
$$

Donde:

$\mathrm{n}=$ Tamaño de la muestra

$\mathrm{N}=$ Numero de población

$Z=$ Puntuación $Z$ del nivel de confianza deseado $\mathrm{e}=$ Margen de error

$\mathrm{p}=$ variabilidad positiva

$\mathrm{q}=$ variabilidad negativa

$$
\begin{gathered}
n=\frac{\frac{(1.65)^{2} * 0.5(1-0.5)}{(0.10)^{2}}}{1+\left(\frac{(1.65)^{2} * 0.5(1-0.5)}{(0.10)^{2} 6290}\right)} \\
n=67
\end{gathered}
$$

Lo que representa ese valor es que, si se toma una muestra de 67 empresas, el $90 \%$ de las veces el dato estará en el intervalo $\pm 10 \%$ respecto al dato observado en la encuesta, para la selección de la muestra se utilizó muestreo aleatorio simple. 


\section{Resultados:}

\section{Análisis vertical}

\section{Análisis por tipo de empresa}

\section{Tabla 4}

Análisis por tipo de empresa

\begin{tabular}{|c|c|}
\hline Empresas de servicios & Principales activos \\
\hline Cuentas por cobrar & $30 \%-50 \%$ \\
\hline Inversiones & $10 \%-20 \%$ \\
\hline Activos Fijos & $10 \%-30 \%$ \\
\hline
\end{tabular}

Fuente: Elaboración propia.

\section{Tabla 5}

Análisis vertical del activo 2016

\begin{tabular}{|c|c|c|}
\hline Principales Activos & $\mathbf{\$}$ & \% Total \\
\hline Cuentas por cobrar & 23340,55 & $11,89 \%$ \\
\hline Inversiones & 0,00 & $0,00 \%$ \\
\hline Activos Fijos & 97203,57 & $49,52 \%$ \\
\hline Total activo & 196289,67 & $100 \%$ \\
\hline
\end{tabular}

Fuente: Elaboración Propia

Interpretación y análisis:

En el año 2016 las empresas del sector de Servicios en sus cuentas por cobrar representan el $11,89 \%$, no tiene inversiones, en activos fijos con el $49,52 \%$ del total activo, por lo tanto la empresa tiene un mayor porcentaje en activos fijos lo que significa que tiene que disminuir en dichos activos debido a que en cuentas por cobrar, inversiones no está dentro del rango estimado para este sector.

Tabla 6

Análisis vertical activo año 2017

\begin{tabular}{|c|c|c|}
\hline Principales Activos & $\$$ & \%Total \\
\hline Cuentas por cobrar & 34945,61 & $13,41 \%$ \\
\hline Inversiones & 40638,83 & $15,59 \%$ \\
\hline Activos Fijos & 120566,82 & $46,26 \%$ \\
\hline Total activo & 260601,44 & $100 \%$ \\
\hline
\end{tabular}

Fuente: Elaboración propia

Paredes Venegas, Cristina Alexandra; Chicaiza Chicaiza, Bryan Leandro \& Ronquillo Llundo, Joselyn Tatiana 


\section{Interpretación y análisis:}

En el año 2016 las empresas del sector de Servicios en sus cuentas por cobrar representan el $13,41 \%$, inversiones con $15,59 \%$, en activos fijos con el 46,26\% del total activo, por lo tanto la empresa tiene un mayor porcentaje en activos

\section{Tabla 7}

Análisis vertical del pasivo y patrimonio 2016 fijos lo que significa que deberían optar por inversiones aumentar sus ventas para que las cuentas por cobrar estén bajo en rango estimado que debería estar constituidas las empresas de este sector.

\begin{tabular}{|c|c|c|}
\hline Pasivo corriente & $\mathbf{5 8 7 9 5 , 0 7}$ & $\mathbf{6 3 , 0 1 \%}$ \\
\hline Pasivo no corriente & 34374,48 & $36,84 \%$ \\
\hline Patrimonio & 143,10 & $0,15 \%$ \\
\hline Total, pasivo y patrimonio & 93312,65 & $100,00 \%$ \\
\hline
\end{tabular}

Fuente: Elaboración propia

Interpretación y análisis:

En el año 2016 las empresas de servicio representan un $63,01 \%$ en pasivo corriente, el
$36,84 \%$ en pasivo no corriente y el $0,15 \%$ en patrimonio del total pasivo más patrimonio, por lo tanto la participación en pasivos es alta.

\section{Tabla 8}

Análisis vertical del pasivo y patrimonio 2017

\begin{tabular}{|c|c|c|}
\hline Pasivo corriente & $\mathbf{7 6 7 6 7 , 5 4}$ & $\mathbf{3 9 , 9 8 \%}$ \\
\hline Pasivo no corriente & 24145,45 & $12,57 \%$ \\
\hline Patrimonio & 91099,15 & $47,44 \%$ \\
\hline Total pasivo y patrimonio & 192012,13 & $100,00 \%$ \\
\hline
\end{tabular}

Fuente: Elaboración propia

En el año 2016 las empresas de servicio representan un $39,98 \%$ en pasivo corriente, el $12,57 \%$ en pasivo no corriente y el $47,44 \%$ en patrimonio del total pasivo más patrimonio, por lo tanto existe una mayor participación del pasivo corriente es alta en comparación con los pasivos no corrientes, además su patrimonio tiene un incremento. 
Tabla 9

Análisis vertical Estado de Resultado

\begin{tabular}{|c|c|c|c|c|}
\hline Año & $\mathbf{2 0 1 6}$ & $\mathbf{2 0 1 7}$ & $\mathbf{( \% ) 2 0 1 6}$ & $\mathbf{( \% ) 2 0 1 7}$ \\
\hline Ventas & 347600,43 & 1140486,22 & 100,00 & 100,00 \\
\hline Costo de ventas & 105562,40 & 56590,02 & 30,37 & 4,96 \\
\hline Gastos & 87128,82 & 79061,25 & 25,07 & 6,93 \\
\hline Utilidad neta & 7812,06 & 7089,75 & 2,25 & 0,62 \\
\hline Perdida del ejercicio & 2671,60 & 1562,60 & 0,77 & 0,14 \\
\hline
\end{tabular}

Fuente: Elaboración propia

En el año 2016, en el sector de servicios se vendió \$347600,43 lo que representa el (100\%), los costos de ventas fueron de $\$ 105562,40$ con el $(30,37 \%)$, los gastos de $\$ 87128,82$ con el $(25,07 \%)$, la utilidad neta de $\$ 7812,06$ con $(2,25 \%)$ y las empresas que registraron pérdida de $\$ 2671,60$ con el $(0,77 \%)$ en promedio.

En el año 2017, en el sector de servicios se vendió \$1140486,22 que lo que representa el (100\%), los costos de ventas fueron de $\$ 56590,02$ con el $(4,96 \%)$, los gastos de $\$ 79061,25$ con el $(6,93 \%)$, la utilidad neta de $\$ 7089,75$ con $(0,62 \%)$ y las empresas que registraron pérdida de $\$ 1562,60$ con el $(0,14 \%)$ en promedio.

\section{Análisis horizontal en los balances generales del 2016-2017}

En el sector de servicios, la variación de los activos corrientes se obtuvo una variación de $\$ 8131,06$ en términos relativos fue el $8,04 \%$ es decir genera efectivo, por otra parte los activos no corrientes tienen una variación absoluta de $\$ 64002,08$ lo que representa una variación relativa del $65,84 \%$ por lo que se puede decir que hubo inversión significativa, así también el pasivo corriente obtuvo una variación absoluta de $\$ 17972,47$ lo que representa un $30,57 \%$ de variación relativa, es decir el sector genera varios financiamientos a corto plazo, de igual forma en el pasivo no corriente se encuentra una disminución de $(\$-10229,03)$ lo cual en termino de porcentaje significa $(-29,76 \%)$, por último el resultado del ejercicio obtuvo una variación absoluta de $(\$-1825,26)$ en términos relativos representa el $(-37,23 \%)$, el sector de servicios disminuyo sus pérdidas a comparación de años anteriores.

\section{Análisis horizontal en los estados de resultado del 2016-2017}

En el sector de servicios, se obtuvo una variación absoluta en total ingresos (actividades ordinarias, otros ingresos) de $\$ 829489,54$ lo que generó una variación relativa de $265,94 \%$ es decir a comparación del año 2016 los ingresos en este sector incrementaron de una manera significativa. Por otra parte, el costo de venta fue de $(\$-48972,37)$ con una variación relativa de $(-46,39 \%)$ lo que significa que se disminuyeron los costos de manera significativa con relación al año 2016. De igual forma en los gatos se obtuvo una variación absoluta de $(\$-8067,57)$ lo que representa un $(-9,26 \%)$ en variación relativa, es decir se disminuyó los gastos incurridos para la prestación de servicios. Por último se obtuvo una

Paredes Venegas, Cristina Alexandra; Chicaiza Chicaiza, Bryan Leandro \& Ronquillo Llundo, Joselyn Tatiana 
disminución en la utilidad en el año 2017 con una variación relativa de $(-9,25 \%)$ lo que es igual a (\$-722,32), así también se obtuvo perdidas las que disminuyeron significativamente entre el año 2016 y 2017 esto representa un $(-41,51 \%)$.

\section{Indicadores financieros}

\section{Indicadores de liquidez 2017}

\section{- Razón corriente}

Razón corriente $=\frac{\text { Activo corriente }}{\text { pasivo corriente }}=\frac{109238,99}{76767,54}$

Se observa que las empresas de servicios tienen una razón corriente de 1,42 en el año 2017 lo que significa que por cada $\$ 1$ que las empresas deben a corto plazo, cuenta con $\$ 1,42$ para respaldar esa obligación.

\section{- Prueba ácida}

$$
\begin{aligned}
\text { prueba ácida }= & \frac{\text { Activo corriente }- \text { inventarios }}{\text { pasivo corriente }} \\
& =\frac{109238,99-8782,83}{76767,54}
\end{aligned}
$$$$
\text { prueba ácida }=1,31
$$

El indicador muestra que las empresas presentan una prueba ácida de 1,31 veces en el año 2017 es decir que por cada \$1 que se debe a corto plazo se cuenta para su cancelación con 1,31 dólares en activos corrientes sin tener que recurrir a la venta de inventarios.

\section{- Capital Neto de trabajo}

Capital neto de trabajo=activo corriente - pasivo corriente capital neto de trabajo $=109238,99$ $76767,54=\$ 32471,45$
Indica que el \$109238,99 de las empresas de servicios tiene invertidos en el activo corriente, $\$ 32471,45$ pueden ser financiados con recursos no corrientes como pasivo de largo plazo o patrimonio.

\section{Indicadores de actividad}

\section{- Rotación de cartera}

rotación de cartera $=\frac{\text { cuentas por cobrar promedio } \times 365 \text { dias }}{\text { ventas }}$ rotación de cartera $=\frac{29143,08 \times 365}{1140486,22}=9,33$ días

En promedio las empresas de servicios tardan 10 días en recuperar su cartera o cuentas por cobrar clientes, es decir la totalidad de la cartera se convierte en efectivo cada 10 días.

\section{- Rotación de inventarios}

rotación de inventarios $=\frac{\text { inventario promedio } \times 365 \text { dias }}{\text { costo de la prestacion de servicio }}$ rotación de inventarios $=\frac{6969,26 \times 365}{56590,02}=44,95$ días

En promedio las empresas de servicios tardan 45 días en convertir su inventario en efectivo o cuentas por cobrar

\section{- Ciclo de efectivo}

ciclo de efectivo $=$ rotación de cartera + rotación de inventarios - rotación de proveedores ciclo de efectivo $=10+45-0=\mathbf{5 5}$ días

Indica que las empresas de servicios presentan un ciclo de efectivo de 55 días es decir que se demoran 55 días desde el momento en que se aporta $\$ 1$ al proceso hasta que lo recupera otra vez. 


\section{- Rotación del activo total}

rotación del activo total $=\frac{\text { ventas }}{\text { activo total promedio }}$ rotación del activo total $=\frac{1140486,22}{228445,56}=4,99$ veces

Los activos totales de las empresas rotaron 4,99 veces en el año, es decir, por cada \$1 invertido en activos totales, las empresas venden $\$ 4,99$ al año.

\section{Indicadores de rentabilidad}

\section{- Margen bruto de utilidad}

margen bruto de utilidad $=\frac{\text { utilidad bruta }}{\text { ventas netas }}$

margen bruto de utilidad $=\frac{1083896,20}{1140486,22}=\mathbf{9 5 , 0 4} \%$

Las ventas generaron $95,04 \%$ de utilidad bruta, es decir, que por cada $\$ 1$ vendido se genera $\$ 95,04$ de utilidad menos los costos de la mercancía vendida.

\section{- Margen operacional}

$$
\begin{aligned}
& \text { margen operacional }=\frac{\text { utilidad operacional }}{\text { ventas netas }} \\
& \text { margen operacional }=\frac{1004834,95}{1140486,22}=\mathbf{8 8 , 1 1} \%
\end{aligned}
$$

La utilidad operacional es del $88,11 \%$ de las ventas netas y por cada $\$ 1$ vendido en el año 2017 se obtuvieron $\$ 88,11$ de utilidad operacional menos los costos y gastos de operación.

\section{- Margen neto de utilidad}

margen neto de utilidad $=\frac{\text { utilidad neta }}{\text { ventas netas }}$

margen neto de utilidad $=\frac{5527,15}{1140486,22}=\mathbf{0 , 4 8} \%$
La utilidad neta correspondió al 0,48\% de las ventas netas del año 2017 es decir que por cada $\$ 1$ vendido generó 0,48 centavos de utilidad neta menos costos, gastos operacionales y no operacionales.

\section{- Rendimiento del patrimonio}

rendimiento del patrimonio $=\frac{\text { utilidad neta }}{\text { patrimonio neto }}$

rendimiento del patrimonio $=\frac{5527,15}{183609,31}=3,01 \%$

La utilidad neta corresponde al 3,01\% sobre el patrimonio durante el año 2017 esto quiere decir que los dueños de los negocios obtuvieron un rendimiento de inversión del $3,01 \%$ en el periodo mencionado.

\section{- Rendimiento del activo total}

rendimiento del activo total $=\frac{\text { utilidad neta }}{\text { activo total promedio }}$
rendimiento del activo total $=\frac{5527,15}{228445,56}=\mathbf{2 , 4 2} \%$

La utilidad neta es del 2,42\% del activo total es decir que por cada $\$ 1$ invertido en activo total se generan $\$ 2,42$ de utilidad neta en el periodo.

\section{- EBITDA}

EBITDA $=$ utlidad operaacional + gasto por depreciacion + gastos por amortizaciones $\mathrm{EBITDA}=1004834,95+925,34+13,81=$ 1005774, 10

\section{Interpretación}

El valor de $\$ 925,34$ corresponde al gasto por depreciación del ejercicio en el año 2017,

Paredes Venegas, Cristina Alexandra; Chicaiza Chicaiza, Bryan Leandro \& Ronquillo Llundo, Joselyn Tatiana 
el valor de $\$ 13,81$ corresponde al gasto por amortización de diferidos significa que aunque la utilidad operacional reportadas por las empresas realmente disponían de $\$ 1005774,10$ en términos de caja.

\section{Indicadores de endeudamiento}

\section{Indicadores de Endeudamiento 2016}

Nivel de endeudamiento $=\frac{\text { Total pasivo }}{\text { Total activo }}=\frac{93287,54}{196289,67}$

Nivel de endeudamiento $=\mathbf{4 7 , 5 3} \%$

El endeudamiento es del 47,53\%, es decir que por cada $\$ 1$ de inversión en activos, 47,53 centavos son financiados por los acreedores como bancos, proveedores, empleados, entre otros. Por lo tanto los acreedores son dueños del 47,53\% y los accionistas del 52,

\section{Concentración de pasivos a corto plazo 2016}

Concentración de pasivo en el corto plazo $=$

$$
\frac{\text { Pasivo corriente }}{\text { Pasivo total }}=\frac{58795,07}{93287,54}
$$

Concentración de pasivo en el corto plazo $=$ $63,03 \%$

La concentración de pasivos en el corto plazo en dichas empresas es del $63,03 \%$, por cada $\$ 1$ de deuda, 63,03centavos tienen vencimiento corriente, además el $63,03 \%$ de los pasivos tienen vencimiento a menos de un año.

\section{Endeudamiento financiero}

Endeudamiento financiero $=$

$\frac{\text { Obligaciones financieras promedio }}{\text { Ventas }}=\frac{3989,04}{1140486,22}$

Endeudamiento financiero $=\mathbf{0 , 3 5 \%}$
El endeudamiento financiero en las empresas de servicio es del $0,35 \%$, es decir, que por cada \$1 que la empresa vende, debe 0,35 centavos al sistema financiero.

\section{Impacto de la carga financiera 2016}

Impacto de la carga financiera $=$

$$
\frac{\text { Gastos financieros }}{\text { Ventas }}=\frac{64,83}{347600,43}
$$

Nivel de endeudamiento $=\mathbf{0 , 0 2 \%}$

El impacto financiero es del $0,02 \%$, o sea, que por cada $\$ 1$ que la empresa vende, se dedica 0,02 a pagar intereses a las instituciones financieras.

\section{Cobertura intereses I 2016}

Cobertura intereses $\mathrm{I}=$

$$
\frac{\text { Utilidad de operación }}{\text { Gastos financieros }}=\frac{154909,21}{64,83}
$$

\section{Cobertura intereses $I=\mathbf{2 3 8 9 , 4 7}$ veces}

Las compañías de servicios durante el periodo obtuvieron una utilidad operacional de 2389,47 veces, por lo tanto, se tiene una capacidad de endeudamiento mayor.

\section{Cobertura de intereses II 2016}

Cobertura intereses II $=$

$$
=\frac{\text { Ebidta }}{\text { Gastos financieros }}=\frac{1005774,10}{64,83}
$$

Cobertura intereses $I I=\mathbf{1 5 5 1 4 , 0 2}$ veces

Las compañías de servicios generan en efectivo durante el periodo, una cantidad 
15514,02 veces superior a los intereses pagados. Es decir, que dispone suficiente efectivo para pagar intereses superiores a los actuales, lo que significa que cuenta con una capacidad de endeudamiento mayor.

\section{Indicadores de Endeudamiento 2017}

Nivel de endeudamiento $=$

$$
\frac{\text { Total pasivo }}{\text { Total activo }}=\frac{101030,97}{260601,44}
$$

Nivel de endeudamiento $=\mathbf{3 8 , 7 7 \%}$

El endeudamiento de las empresas de servicios es de $38,77 \%$, que por cada $\$ 1$ que tienen invertido en activos, 38,77 centavos son financiados por los acreedores, como bancos, proveedores, empleados, entre otras. Por lo tanto, los acreedores son dueños del 38,77\%, de las empresas y los accionistas del 61,23\%.

\section{Concentración de pasivos a corto plazo 2017}

Concentración de pasivo en el corto plazo $=$

$$
: \frac{\text { Pasivo corriente }}{\text { Pasivo total }}=\frac{76767,54}{101030,97}
$$

Concentración de pasivo en el corto plazo $=$ $\mathbf{7 5 , 9 8 \%}$

La concentración de pasivos en el corto plazo es de $75,98 \%$ por cada $\$ 1$ de deuda 75,98 centavos tienen vencimiento corriente., además el $75,98 \%$ de los pasivos tienen vencimiento a menos de un año.

\section{Impacto de la carga financiera 2017}

Impacto de la carga financiera $=$

$$
\frac{\text { Gastos financieros }}{\text { Ventas }}=\frac{272,38}{1140486,22}
$$

Nivel de endeudamiento $=\mathbf{0 , 0 2} \%$

Este indicador muestra que el impacto financiero es del $0,02 \%$, o sea, que por cada $\$ 1$ que la empresa vende, dedica 0,02 a pagar intereses a las instituciones financieras.

\section{Cobertura intereses I 2017}

$$
\text { Cobertura intereses } \mathrm{I}=
$$

$$
\frac{\text { Utilidad de operación }}{\text { Gastos financieros }}=\frac{1004834,95}{272,38}
$$

Cobertura intereses $I=\mathbf{3 6 8 9 , 0 9}$ veces

Las compañías generan durante el periodo, una utilidad operacional igual a 3689,09 veces los intereses pagados, es decir, que dispone de una capacidad suficiente para intereses superiores a los actuales.

\section{Cobertura de intereses II 2016}

$$
\text { Cobertura intereses II }=
$$

$$
\frac{\text { Ebidta }}{\text { Gastos financieros }}=\frac{1005774,10}{272,38}
$$

\section{Cobertura intereses $\mathrm{II}=\mathbf{3 6 9 2 , 5 4}$ veces}

Las compañías de servicios generan efectivo, una cantidad 3292,54 veces superior a los intereses pagados. Es decir, lo cual significa que, cuenta con una capacidad de endeudamiento mayor.

\section{Conclusión}

El análisis financiero permite detectar puntos débiles y fuertes de las empresas en un determinado período, también la variación de un período a otro, esto permite tomar decisiones de inversión, operativas, y de financiamiento en

Paredes Venegas, Cristina Alexandra; Chicaiza Chicaiza, Bryan Leandro \& Ronquillo Llundo, Joselyn Tatiana 
base a los resultados obtenidos al momento de aplicarlos.

Los Indicadores Financieros yeconómicos se los realizan a través de la información contable, estos nos ayudan a conocer el movimiento de las distintas cuentas que maneja una empresa lo que nos permite realizar un completo análisis.

Existe un sinnúmero de indicadores financieros y económicos de acuerdo con el tipo de empresa, además son expresados en términos de razones, por otra parte, es necesario conocer a fondo la actividad a la que se dedica la entidad para una adecuada aplicación de indicadores.

\section{Referencias bibliográficas}

Baena, G. (2014). Metodología de la Investigación. Grupo Editorial Patria. México. Recuperado de: http://www.editorialpatria.com.mx/ pdffiles/9786074384093.pdf

Barrera, A. \& Jiménez, A. (2017). Caracterización del sector de servicios a las empresas en Manizales. Recuperado de: http://investinmanizales. org/wp-content/uploads/2017/03/ Caracterizaci $\%$ C $3 \%$ B $3 n-$ sectorservicios.pdf

Bravo, J. (2017). Factores que afectan la sostenibilidad y el crecimiento de las pequeñas empresas en la ciudad de Bucaramanga - Santander - Colombia. Sigma, 4(1), 65-85

Morales, O. (2014). Fundamentos de la Investigación. Recuperado de: http:// webdelprofesor.ula.ve/odontologia/ oscarula/publicaciones/articulo18.pdf

Núñez, F. (2015). Análisis Económico y Financiero de la empresa de transportes Expreso Internacional Tour Perú S.R.L Periodos 2013 - 2014. Universidad Nacional del Altiplano, Puno-Perú. Recuperado de: http://repositorio.unap. edu.pe/bitstream/handle/UNAP/2382/ Nu\%C3\%B1ez_Ramos_Fernando_ Edwing.pdf? sequence $=1 \&$ isAllowed $=y$

Galán,L.;Castro,M.\&Pérez,L.(2016).Aplicación Del Modelo De Análisis Financiero En Las Mipymes Comercializadoras De Ocotlán, Jalisco. Recuperado de: http:// www.web.facpya.uanl.mx/vinculategica/ Revistas/R2/3709\%20-\%203735\%20 Apliacion\%20del\%20Modelo.pdf

Hernández, R.; Fernández, C. \& Baptista, P. (2006). Metodología de la investigación(Vol. 3). México: McGrawHill.

Herrera, G. (2014). Importancia del sector terciario: la tercerización de la Economía. Recuperado de: http://www.iesgaherrera. com/files/GEOGRAF\%C3\%8DA\%20 (BachilleratB)/Importancia_del_sector_ terciario_2014.pdf

Ibarra, A. (2009). Desarrollo del Análisis Factorial Multivariable Aplicado al Análisis Financiero Actual. Colombia. Recuperado de: http://www.eumed.net/ librosgratis/2010a/666/ALGUNOS\%20 ANTECEDENTES\% $\%$ 20SOBRE $\% 20$ LA\%20EVOLUCION\%20DE\%20 RATIOS.htm 
Instituto Nacional de Estadísticas y Censos (2016). Directorio De Empresas $Y$ Establecimientos. Quito, Ecuador Recuperado de: http://www. ecuadorencifras.gob.ec/documentos/ webinec/Estadisticas_Economicas/ DirectorioEmpresas/Directorio_ Empresas_2016/Principales_Resultados_ DIEE_2016.pdf

Madrigal, F.; Ayala, S. \& Chávez, L. (2015). Análisis e interpretación de estados financieros en condiciones de incertidumbre. Revista Administración \& Finanzas. Recuperado de: http://www. ecorfan.org/bolivia/researchjournals/ Administracion_y_Finanzas/vol2num2/ Administracion_Finanzas_Vol2Num2_ Final_12.pdf

Panchi, V. (2017). Una mirada del proceso de regulación contable internacional en el contexto de globalización. Sigma, 4(1), 115-129

Prieto, C. (2010). Análisis Financiero. Fundación para la Educación Superior San Mateo. Bogotá D.C. Colombia. Recuperado de: https://www.sanmateo .edu.co/ documentos/publicacion-analisisfinanciero.pdf

Puerta, F.; Vergara, J. \& Huertas, N. (2018). Análisis Financiero Desarrollar en su Evolución. Criterio Libre. Vol. 16. No. 28. Bogotá, Colombia. Recuperado de: https://www.researchgate.net/ publication/327193736_Analisis_ financiero_enfoques_en_su_evolucion
Superintendencia de Compañías (s.f). Tabla de indicadores. Quito, Ecuador. Recuperado de: http://reporteria.supercias.gob.ec/ portal/samples/images/docs/tabla_ indicadores.pdf

Superintendencia de Compañías y Seguros (s.f). Portal de Información. Ecuador Recuperado de: http://appscvs.supercias. gob.ec/portalInformacion/sector societario.zul

Torres, E. (2006). Evolución del Empleo en el Sector Terciario en Venezuela. Mérida, Venezuela Recuperado de: https://www. redalyc.org/pdf/4655/465545874005.pdf

Torres, M. (2014). Introducción a los Indicadores Financieros. Recuperado de: https://prezi.com/gdkd_opz33jp/ introduccion-a-los-indicadoresfinancieros/

Vargas, R. (2008). Estadística II. Bogotá, Colombia. Recuperado de: http:// www.esap.edu.co/portal/wp-content/ uploads/2017/10/6-Estadstica-ii.pdf

Zhanay, M. (2013). Análisis e interpretación de los Estados Financieros de la Cooperativa de taxis La Pradera de la Ciudad de Loja. Universidad Nacional de Loja, Loja-Ecuador. Recuperado de: $\quad$ http://dspace.unl.edu.ec/jspui/ bitstream/123456789/1706/1/TESIS\%20 $\% 20$ DEFINITIVA.pdf

Paredes Venegas, Cristina Alexandra; Chicaiza Chicaiza, Bryan Leandro \& Ronquillo Llundo, Joselyn Tatiana 